Research Paper

\title{
Risk Factors for Oxaliplatin-Induced Hypersensitivity Reactions in Japa- nese Patients with Advanced Colorectal Cancer
}

\author{
Kyoko Seki 1, Kenzou Senzaki 1, Yasuo Tsuduki 2, Takeshi loroi ${ }^{3}$, Michiko Fujii 4, Hiroko Yamauchi ${ }^{5}$, \\ Yukinari Shiraishi ${ }^{1}$, Izumi Nakata ${ }^{2}$, Kohshi Nishiguchi ${ }^{3}$, Teruhisa Matsubayashi ${ }^{4}$, Yoshihide Takakubo ${ }^{5}$, \\ Noboru Okamura ${ }^{6}$, Motohiro Yamamori ${ }^{6}$, Takao Tamura ${ }^{7}$ and Toshiyuki Sakaeda 8 凶 \\ 1. Department of Pharmacy, Japan Labour Health and Welfare Organization, Kobe Rosai Hospital, Kobe 651-0053, Japan \\ 2. Department of Pharmacy, National Hospital Organization Kobe Medical Center, Kobe 654-0155, Japan \\ 3. Department of Pharmacy, Kobe University Hospital, Kobe 650-0017, Japan \\ 4. Department of Pharmacy, Japanese Red Cross Kobe Hospital, Kobe 651-0073, Japan \\ 5. Department of Pharmacy, Shinko Hospital, Kobe 651-0072, Japan \\ 6. School of Pharmacy and Pharmaceutical Sciences, Mukogawa Women's University, Nishinomiya 663-8179, Japan \\ 7. Department of Medical Oncology, Kinki University Nara Hospital, Nara 630-0293, Japan \\ 8. Graduate School of Pharmaceutical Sciences, Kyoto University, Kyoto 606-8501, Japan
}

Corresponding author: Toshiyuki Sakaeda, Ph.D., Center for Integrative Education of Pharmacy Frontier (Frontier Education Center), Graduate School of Pharmaceutical Sciences, Kyoto University 46-29 Yoshidashimoadachi-cho, Sakyo-ku, Kyoto 606-8501, Japan. Tel: +81-75-753-9560, Fax: +81-75-753-4502, E-Mail: sakaedat@pharm.kyoto-u.ac.jp

( ) Ivyspring International Publisher. This is an open-access article distributed under the terms of the Creative Commons License (http://creativecommons.org/ licenses/by-nc-nd/3.0/). Reproduction is permitted for personal, noncommercial use, provided that the article is in whole, unmodified, and properly cited.

Received: 2010.06.17; Accepted: 2011.03.04; Published: 2011.03.10

\begin{abstract}
Objective: Previously, we suggested that oxaliplatin (L-OHP)-related grade 3/4 hypersensitivity reactions occurred immediately after the initiation, but grade $\mathrm{I} / 2$ reactions did not. This study was conducted to clarify the risk factors for L-OHP-related hypersensitivity reactions.

Methods: Clinical data from 108 Japanese patients with colorectal cancer were analyzed, who were treated with L-OHP-containing regimens, FOLFOX4 and/or mFOLFOX6. The risk factors examined included demographic data, preexisting allergies, laboratory test data, treatment regimen, treatment line of therapy, pretreatment with steroids, total number of cycles and cumulative amount of L-OHP.

Results: The incidence of grade $\mathrm{I} / 2$ and grade $3 / 4$ hypersensitivity reactions were found at $13.0 \%(14 / 108)$ and $9.3 \%(10 / 108)$, respectively. Female $(P=0.037)$, preexisting allergies $(P=0.004)$ and lower level of lactate dehydrogenase $(P=0.003)$ were risk factors for grade $I / 2$ hypersensitivity reactions, and higher neutrophil count $(P=0.043)$ and lower monocyte count $(P=0.007)$ were for grade $3 / 4$ reactions. Total number of cycles were larger in the patients with grade $3 / 4$ reactions than those without reactions $(P=0.049)$.

Conclusions: Further extensive examination with a large number of patients is needed to establish a patient management strategy.
\end{abstract}

Key words: colorectal cancer, FOLFOX, oxaliplatin (L-OHP), hypersensitivity reactions, risk factor

\section{Introduction}

The treatment of metastatic colorectal cancer has progressed significantly over the past 20 years. In the early 1990s, repetitive injections of a bolus of 5-fluorouracil (5-FU) and leucovorin (LV) were the standard treatment, preferably with the RPMI regimen [1] or Mayo Clinic regimen [2]. In the late 1990s, clinical outcome was improved with the continuous infusion of 5-FU, and the LV5FU2 regimen consisting 
of a bolus injection of 5-FU and infusion of 5-FU/LV resulted in a median survival time (MST) of 14.7 months in first-line therapy $[3,4]$. Treatment has since progressed remarkably with the development of the anticancer drugs irinotecan (CPT-11) and oxaliplatin (L-OHP). Although only a slight improvement in clinical outcome was obtained with a combination of bolus 5-FU/LV and CPT-11, known as the IFL regimen [5], the FOLFIRI regimen consisting of a bolus injection of 5-FU, CPT-11 and infusion of 5-FU/LV has increased MST to 17.4 months [6,7]. The simultaneously developed FOLFOX regimen consisting of a bolus injection of 5-FU, L-OHP and infusion of 5-FU/LV was also promising, with a MST of 16.2-19.5 months $[4,7,8]$. Currently, the FOLFIRI or FOLFOX regimen, with or without a targeted monoclonal antibody, is the standard treatment [9-12], and future improvements will likely require the incorporation of or substitution with a novel anticancer drug, personalization based on genetic profiling, or pharmacokinetically-guided administration.

Hypersensitivity reactions are a well-established complication of the platinum agents, cisplatin and carboplatin [13-16]. L-OHP, a third-generation platinum agent, has been increasingly recognized to cause hypersensitivity reactions, but the incidence still varies in reports [17-23], and little information is available for the risk factors and therefore their management, especially in severe cases. Previously, we suggested that grade $3 / 4$ hypersensitivity reactions occurred immediately after the initiation, but in contrast, grade $1 / 2$ reactions did not [24]. This multicenter retrospective study was conducted to clarify the risk factors for L-OHP-related hypersensitivity reactions. Clinical data from patients who experienced hypersensitivity reactions were compared to those from patients who did not. The risk factors examined included demographic data, preexisting allergies, laboratory test data, treatment regimen, treatment line of therapy, pretreatment with steroids, total number of cycles and cumulative amount of L-OHP.

\section{Patients and Methods \\ Eligibility}

All patients were treated with the FOLFOX4 and/or mFOLFOX6 regimens at either of Labor Health and Welfare Organization Kobe Rosai Hospital, National Hospital Organization Kobe Medical Center, Kobe University Hospital, Kobe Red Cross Hospital, and Shinko Hospital, Japan, from April 2005 to March 2009. All patients had histologically or cytologically confirmed advanced or metastatic colorectal adenocarcinoma. Patients had received no prior chemotherapy or only one regimen with a washout period of more than 4 weeks after the final day of the previous treatment. Adjuvant chemotherapy performed more than 6 months previously was not counted as previous treatment. Further eligibility criteria included: 1) age of 20-75 years; 2) Eastern Cooperative Oncology Group (ECOG) performance status of 0 or 1 ; 3) life expectancy of 3 months or more; 4) adequate hematological (leukocyte count: $4,000 / \mathrm{mm}^{3}-12,000 / \mathrm{mm}^{3}$, neutrophil count: $2,000 / \mathrm{mm}^{3}$ or more, platelets: $100,000 / \mathrm{mm}^{3}$ or more), hepatic (transaminases: 2.5 times or less of the upper limit of normal, total bilirubin: $2.0 \mathrm{mg} / \mathrm{dL}$ or less), and renal (serum creatinine: less than the upper limit of normal) function; and 5) ability to take oral medication. Depending on the clinical situation, patients who did not meet the criteria can be treated with L-OHP under the careful supervision of medical doctors. Patients were excluded, if they had either brain metastases, a history of other neoplasms (except for cured nonmelanoma skin carcinoma or cured carcinoma in situ), a history of severe drug allergies, interstitial pneumonitis or pulmonary fibrosis, severe pleural effusion or ascites, active infection, bowel obstruction, diarrhea, and serious uncontrolled comorbidity or medical conditions. Pregnant or lactating women or women not using an effective contraception were also excluded. This retrospective study was approved by institutional review boards of each of the 5 hospitals.

\section{Data Analysis}

Hypersensitivity reactions were assessed and classified according to the National Cancer Institute Common Criteria (NCI-CTCAE v3.0). Clinical data were compared between the patients who experienced hypersensitivity reactions and those who did not. The risk factors examined included gender, age, height, weight, performance status, and preexisting allergies (allergy for specific food or drug, pollinosis or allergic rhinitis). The effects of laboratory test data on one day before or on the day of the start of therapy were also analyzed, including erythrocyte count, hemoglobin, hematocrit, leukocyte count, neutrophil count, lymphocyte count, eosinophil count, basophil count, monocyte count, platelet count, aspartate aminotransferase (AST), alanine aminotransferase (ALT), gamma-glutamyl transpeptidase ( $\gamma-G T P)$, total bilirubin (T-Bil), alkaline phosphatase (ALP), lactate dehydrogenase $(\mathrm{LDH})$, blood urea nitrogen (BUN), serum creatinine (Scr), carcinoembryonic antigen (CEA) and CA19-9 antigen (CA19-9). Treatment regimen, treatment line of therapy, pretreatment with steroids, total number of cycles and cumulative 
amount of L-OHP were also examined in terms of susceptibility to hypersensitivity reactions.

\section{Statistical Analysis}

All values reported are the mean \pm standard deviation (SD). The unpaired Student's $t$-test/Welch's test or Mann-Whitney's $U$ test was used for two-group comparisons of the values. Fisher's exact test was used for the analysis of contingency tables. $\mathrm{P}$ values of less than 0.05 were considered to be significant.

\section{Results}

Demographics and the data on laboratory test and chemotherapy in 108 patients who received L-OHP are summarized in Table 1 . Average values of age, height and total body weight of 108 patients were $64.5 \pm 9.8$ years, $160.6 \pm 9.0 \mathrm{~cm}$ and $57.1 \pm 9.7 \mathrm{~kg}$, respectively. Ten of 108 patients $(9.3 \%)$ experienced grade $3 / 4$ hypersensitivity reactions, whereas grade
$1 / 2$ events occurred in 14 patients $(13.0 \%)$.

There was no statistical difference of age, height, weight and performance status between the patients with no and grade $1 / 2$ hypersensitivity reactions. Compared with men, woman had a higher susceptibility to grade $1 / 2$ hypersensitivity reactions $(p=0.037)$. Eight of 14 patients $(57.1 \%)$ with grade $1 / 2$ hypersensitivity reactions had preexisting allergies, but only $17.9 \%(15 / 84)$ of patients without reactions $(p=0.004)$. Laboratory test data on hematological, hepatic and renal functions were independent of, but lower LDH level was risk factor for grade $1 / 2$ hypersensitivity reactions $(\mathrm{P}=0.003)$. No meaningful differences were observed between the patients with no and grade $1 / 2$ hypersensitivity reactions, with regards to treatment regimen, treatment line of therapy, pretreatment with steroids, total number of cycles and cumulative amount of L-OHP.

Table I. Demographics, laboratory test and chemotherapy in the patients with no, grade $1 / 2$ and grade $3 / 4$ hypersensitivity reactions

\begin{tabular}{|c|c|c|c|}
\hline Hypersensitivity & $\begin{array}{l}\text { No hypersensitivity } \\
\mathrm{N}=84\end{array}$ & $\begin{array}{l}\text { Grade } 1 / 2 \\
N=14\end{array}$ & $\begin{array}{l}\text { Grade } 3 / 4 \\
N=10\end{array}$ \\
\hline \multicolumn{4}{|l|}{ Patients } \\
\hline Male/female & $56 / 28$ & $5 / 9$ * & $6 / 4$ \\
\hline Age, year & $65.2 \pm 9.2[36-83]$ & $60.1 \pm 12.2[36-72]$ & $65.5 \pm 10.6[46-76]$ \\
\hline Height, $\mathrm{cm}$ & $160.9 \pm 9.0[132.0-179.2]$ & $158.0 \pm 9.7[143.8-168.9]$ & $162.1 \pm 8.4[149.5-174.0]$ \\
\hline Weight, kg & $56.4 \pm 9.8[36.0-80.0]$ & $59.5 \pm 9.4[44.0-76.0]$ & $59.9 \pm 8.7[50.0-75.5]$ \\
\hline Performance status, $0 / 1 / 2 / 3$ & $70 / 6 / 6 / 2$ & $12 / 1 / 1 / 0$ & $9 / 1 / 0 / 0$ \\
\hline Allergic history, yes/no & $15 / 69$ & $8 / 6^{*}$ & $2 / 8$ \\
\hline \multicolumn{4}{|l|}{ Laboratory test } \\
\hline Erythrocyte count, $\times 10^{4} / \mathrm{mm}^{3}$ & $384 \pm 57[225-489]$ & $389 \pm 64[287-491]$ & $424 \pm 57[359-520]$ \\
\hline Leukocyte count, $/ \mathrm{mm}^{3}$ & $6848 \pm 3560[3100-30500]$ & $6086 \pm 1964$ [3370-11300] & $7515 \pm 2509$ [4600-14010] \\
\hline Neutrophil count, $/ \mathrm{mm}^{3}$ & $4639 \pm 3500[839-28975]$ & $3744 \pm 1877$ [1618-8780] & $5938 \pm 2929[3340-12889]$ * \\
\hline Lymphocyte count, / $\mathrm{mm}^{3}$ & $1461 \pm 621[224-3054]$ & $1600 \pm 532[1170-2503]$ & $1357 \pm 412[773-1864]$ \\
\hline Monocyte count \% & $7.6 \pm 2.9[1.0-19.8]$ & $7.8 \pm 1.9[4.7-11.0]$ & $5.2 \pm 1.8[2.0-7.9]$ * \\
\hline Platelet count, $\times 10^{4} / \mathrm{mm}^{3}$ & $28.2 \pm 9.8[13.2-53.3]$ & $24.1 \pm 9.1[10.9-42.9]$ & $31.4 \pm 15.1[14.8-57.0]$ \\
\hline Lactate dehydrogenase, IU/L & $298 \pm 230[8-1248]$ & $171 \pm 29[135-210]$ * & $451 \pm 297$ [146-985] \\
\hline \multicolumn{4}{|l|}{ Chemotherapy } \\
\hline FOLFOX4/mFOLFOX6/both & $41 / 40 / 3$ & $7 / 7 / 0$ & $6 / 3 / 1$ \\
\hline Line of therapy, 1st/2nd or more & $28 / 56$ & $4 / 10$ & $4 / 6$ \\
\hline Pretreatment with steroids, yes/no & $81 / 3$ & $12 / 2$ & $10 / 0$ \\
\hline Total cycle number of therapy & $6.6 \pm 4.0[1-19]$ & $8.4 \pm 4.4[2-17]$ & $9.3 \pm 3.9[5-16]^{*}$ \\
\hline Cumulative amount of L-OHP, $\mathrm{mg} / \mathrm{m}^{2}$ & $521.4 \pm 329.3$ [ 40.8-1374.3 ] & $675.8 \pm 352.2[156.3-1306.1]$ & $726.7 \pm 316.3[406.3-1342.3]$ \\
\hline
\end{tabular}

The values are the mean $\pm S D$ with the range in parentheses.

${ }^{*} \mathrm{P}<0.05$, compared with the patients without hypersensitivity reactions 
As for grade $3 / 4$ reactions, no difference of demographic data was found, when compared with the patients with no reactions. Preexisting allergies were also not predictive of grade $3 / 4$ hypersensitivity reactions. No association was found for the laboratory test data on hepatic and renal functions, but higher neutrophil count $(\mathrm{P}=0.043)$ and lower monocyte count $(\mathrm{P}=0.007)$ were risk factors for grade $3 / 4$ reactions. Treatment-related conditions were independent of grade $3 / 4$ hypersensitivity reactions, except for total cycle number of therapy $(p=0.049)$.

\section{Discussion}

Hypersensitivity reactions to the platinum agents cisplatin and carboplatin are well documented [13-16]. For cisplatin, the incidence of hypersensitivity reactions have been reported as $2-5 \%$ when administered as a single agent and $5-10 \%$ when combined with other agents [14]. Carboplatin induces reactions with an incidence of $12-27 \%$ [13,16]. With the increasing use of L-OHP in clinical practice, L-OHP-induced hypersensitivity reactions have been encountered frequently, and reportedly, the incidence ranged from $3.6 \%$ to $18.9 \%$ in total, but serious reactions hardly happened in Western countries [17-22]. In a randomized phase III trial, the MOSAIC trial, $10.3 \%$ of the 1108 patients experienced hypersensitivity reactions, and $2.3 \%$ and $0.6 \%$ had grade 3 and grade 4 reactions, respectively [17]. In this study, we found that $22.2 \%$ of Japanese patients who were treated with L-OHP-containing regimens experienced hypersensitivity reactions, and grade $3 / 4$ events occurred in $9.3 \%$ of patients. This incidence is relatively high than those in the reports, suggesting a racial effect. However, more recently, a report from Japanese affiliation indicated that $17.0 \%$ of 125 patients experienced hypersensitivity reactions, with grade $3 / 4$ at $4.0 \%$ [23]. These values are still higher than those in the MOSAIC trial, but lower than those in our study. Thus, clinical factors might affect the incidence, including the pre-dosing of antihistamines or steroids.

Only a few investigations have attempted to identify potential risk factors for hypersensitivity reactions to L-OHP. Lee et al. analyzed the possible association between L-OHP-induced anaphylaxis and metastases, but no significant association was identified [25]. Kim et al. suggested that a higher incidence was found in younger patients, female patients, and patients with salvage therapy [26], whereas Shibata et al. reported no correlation with gender and history of allergy [23]. Here, it was suggested that female $(\mathrm{P}=0.037)$, preexisting allergies $(\mathrm{P}=0.004)$ and lower LDH level $(\mathrm{P}=0.003)$ were risk factors for grade $1 / 2$ hypersensitivity reactions, and higher neutrophil count $(\mathrm{P}=0.043)$ and lower monocyte count $(\mathrm{P}=0.007)$ were for grade $3 / 4$ reactions (Table 1 ). The reasons for increase of risk amongst female are unknown, but this finding implicates a possible role of hormonal influences [26]. A history of allergy for specific food or drug, pollinosis or allergic rhinitis were handled as preexisting allergies, and the common mechanisms might exist for these allergies and grade $1 / 2$ hypersensitivity reactions. $\mathrm{LDH}$ is found in the liver, kidneys, striated muscle, skin and heart muscle, and therefore is widely used to diagnose the condition of patients with lung, heart, blood and malignant diseases. The patients in this study were all with colorectal adenocarcinoma and most of them showed higher LDH level than normal levels. Here, the LDH level was within the normal range in the patients with grade $1 / 2$ hypersensitivity reactions (Table 1 ), although the reasons are not clear. Neutrophils and monocyte/macrophages are phagocytic cells, which play an important role in host defense, but are also inflammatory cells, that can mediate tissue damage. Both cells are essential for the innate immune system, but recent researches suggest that the recognition and subsequent engulfment of apoptotic neutrophils by macrophages is involved in the resolution of inflammation [27, 28], and therefore the stage of inflammation in the patients with higher neutrophil count and lower monocyte count is supposed to be different from others. Total cycle number of therapy was larger in the patients with grade $3 / 4$ reactions than those without reactions $(\mathrm{P}=0.049)$, and thus extensive repetition of therapy might result in grade $3 / 4$ reactions (Table 1). Further extensive examination with a large number of patients is needed to identify the risk factors, and to establish a patient management strategy.

Although hypersensitivity reactions are a well-established complication of the platinum agents [13-23], their exact mechanism remains unclear. The agents are thought to induce a type I response mediated by IgE, followed by the release of histamine and cytokines, since reactions usually occur after multiple infusions [29-32]. Recent studies have suggested the involvement of a type IV reaction, i.e., T-cell-mediated production of cytokines, such as tumor necrosis factor-alpha and interleukin-6, especially for cisplatin and carboplatin [29-32]. As far as L-OHP is concerned, most reactions are thought to be of type I, but reports of hemolysis and thrombocytopenia suggest a type II reaction, and chronic urticaria, joint pain and proteinuria can be attributed to a type III reaction [29-32]. In our previous report, we suggested that grade $3 / 4$ hypersensitivity reactions occurred immediately after the initiation, but in contrast, grade $1 / 2$ reactions did not [24]. Here, it was found that the risk factors for 
grade $3 / 4$ reactions were not in accordance with those for grade $3 / 4$ reactions. These findings might suggest that the different mechanisms exist to separate them. Delayed hypersensitivity reactions are generally less severe than acute reactions, and might include redness of the palms and torso, and pruritus [30]. Strategies to manage delayed hypersensitivity reactions include desensitization approaches such as use of steroids, antihistamines, and prolongation of infusion time, but L-OHP discontinuation is recommended for acute anaphylactic reaction [30].

In conclusion, this multicenter retrospective study was conducted to clarify the risk factors for L-OHP-related hypersensitivity reactions. Clinical data from the patients who experienced hypersensitivity reactions were compared to those from the patients who did not. The incidence of grade $1 / 2$ and grade $3 / 4$ hypersensitivity reactions were found at $13.0 \%$ and $9.3 \%$, respectively. Female, preexisting allergies and lower LDH level were risk factors for grade $1 / 2$ hypersensitivity reactions, and higher neutrophil count and lower monocyte count were for grade 3/4 reactions. Extensive repetition of therapy resulted in grade $3 / 4$ reactions. Further extensive examination with a large number of patients is needed to establish a patient management strategy.

\section{Conflict of Interest}

The authors have declared that no conflict of interest exists.

\section{References}

1. Petrelli N, Douglass JrHO, Herrera L, et al. The modulation of fluorouracil with leucovorin in metastatic colorectal carcinoma: a prospective randomized phase III trial. Gastrointestinal Tumor Study Group. J Clin Oncol. 1989; 7: 1419-1426.

2. Poon MA, O'Connell MJ, Wieand HS, et al. Biochemical modulation of fluorouracil with leucovorin: confirmatory evidence of improved therapeutic efficacy in advanced colorectal cancer. J Clin Oncol. 1991; 9: 1967-1972.

3. de Gramont A, Louvet C, André T, et al. A review of GERCOD trials of bimonthly leucovorin plus 5-fluorouracil 48-h continuous infusion in advanced colorectal cancer: evolution of a regimen. Groupe d'Etude et de Recherche sur les Cancers de l'Ovaire et Digestifs (GERCOD). Eur J Cancer. 1998; 34: 619-626.

4. de Gramont A, Figer A, Seymour M, et al. Leucovorin and fluorouracil with or without oxaliplatin as first-line treatment in advanced colorectal cancer. J Clin. Oncol. 2000; 18: 2938-2947.

5. Saltz LB, Cox JV, Blanke C, et al. Irinotecan plus fluorouracil and leucovorin for metastatic colorectal cancer. Irinotecan Study Group. N Engl J Med. 2000; 343: 905-914.

6. Douillard JY, Cunningham D, Roth AD, et al. Irinotecan combined with fluorouracil compared with fluorouracil alone as first-line treatment for metastatic colorectal cancer: a multicentre randomised trial. Lancet. 2000; 355: 1041-1047.

7. Tournigand C, André T, Achille E, et al. FOLFIRI followed by FOLFOX6 or the reverse sequence in advanced colorectal cancer: A randomized GERCOR study. J Clin Oncol. 2004; 22: 229-237.
8. Goldberg RM, Sargent DJ, Morton RF, et al. A randomized controlled trial of fluorouracil plus leucovorin, irinotecan, and oxaliplatin combinations in patients with previously untreated metastatic colorectal cancer. J Clin Oncol. 2004; 22: 23-30.

9. Grothey A, Sargent D, Goldberg RM, et al. Survival of patients with advanced colorectal cancer improves with the availability of fluorouracil-leucovorin, irinotecan, and oxaliplatin in the course of treatment. J Clin Oncol. 2004; 22: 1209-1214.

10. Venook A. Critical evaluation of current treatments in metastatic colorectal cancer. Oncologist. 2005; 10: 250-261.

11. Lee JJ, Chu E. An update on treatment advances for the first-line therapy of metastatic colorectal cancer. Cancer J. 2007; 13: 276-281.

12. Sabharwal A, Kerr D. Chemotherapy for colorectal cancer in the metastatic and adjuvant setting: past, present and future. Expert Rev Anticancer Ther. 2007; 7: 477-487.

13. Shepherd GM. Hypersensitivity reactions to chemotherapeutic drugs. Clin Rev Allergy Immunol. 2003; 24: 253-262.

14. Heywood GR, Rosenberg SA, Weber JS, et al. Hypersensitivity reactions to chemotherapy agents in patients receiving chemoimmunotherapy with high-dose interleukin 2. J Natl Cancer Inst. 1995; 87: 915-922.

15. Markman M, Kennedy A, Webster K, et al. Clinical features of hypersensitivity reactions to carboplatin. J Clin Oncol. 1999; 17: 1141.

16. Markman M. Toxicities of the platinum antineoplastic agents. Expert Opin Drug Saf. 2003; 2: 597-607.

17. Andre $T$, Boni $C$, Mounedji-Boudiaf $L$, et al. Multicenter International Study of Oxaliplatin/5-Fluorouracil/Leucovorin in the Adjuvant Treatment of Colon Cancer (MOSAIC) Investigators, Oxaliplatin, fluorouracil, and leucovorin as adjuvant treatment for colon cancer. N Engl J Med. 2004; 350: 2343-2351.

18. Thomas RR, Quinn MG, Schuler B, et al. Hypersensitivity and idiosyncratic reactions to oxaliplatin. Cancer. 2003; 97: 2301-2307.

19. Siu SW, Chan RT, Au GK, et al. Hypersensitivity reactions to oxaliplatin: experience in a single institute. Ann Oncol. 2006; 17: 259-261.

20. Maindrault-Goebel F, Andre T, Tournigand C, et al. Allergic-type reactions to oxaliplatin: retrospective analysis of 42 patients. Eur J Cancer. 2005; 41: 2262-2267.

21. Gowda A, Goel R, Berdzik J, et al. Hypersensitivity reactions to oxaliplatin: incidence and management. Oncology. 2004; 18: 1671-1675.

22. Brandi G, Pantaleo MA, Galli C, et al. Hypersensitivity reactions related to oxaliplatin (OHP). Br J Cancer. 2003; 89: 477-481.

23. Shibata Y, Ariyama H, Baba E, et al. Oxaliplatin-induced allergic reaction in patients with colorectal cancer in Japan. Int J Clin Oncol. 2009; 14: 397-401.

24. Seki K, Senzaki K, Tsuduki Y, et al. Multicenter trial on hypersensitivity reactions following treatment with FOLFOX regimens. Jpn J Pharm Health Care Sci. 2008; 34: 919-926.

25. Lee MY, Yang MH, Liu JH, et al. Severe anaphylactic reactions in patients receiving oxaliplatin therapy: a rare but potentially fatal complication. Support Care Cancer. 2007; 15: 89-93.

26. Kim BH, Bradley T, Tai J, et al. Hypersensitivity to oxaliplatin: an investigation of incidence and risk factors, and literature review. Oncology. 2009; 76: 231-238.

27. Kantari C, Pederzoli-Ribeil M, Witko-Sarsat V. The role of neutrophils and monocytes in innate immunity. Contrib Microbiol. 2008; 15:118-146.

28. Fox S, Leitch AE, Duffin R, et al. Neutrophil apoptosis: relevance to the innate immune response and inflammatory disease. J Innate Immun. 2010; 2: 216-227.

29. Syrigou E, Syrigos K, Saif MW. Hypersensitivity reactions to oxaliplatin and other antineoplastic agents. Curr Allergy Asthma Rep. 2008; 8: 56-62. 
30. Eng C. Toxic effects and their management: daily clinical challenges in the treatment of colorectal cancer. Nat Rev Clin Oncol. 2009; 6: 207-218.

31. Makrilia N, Syrigou E, Kaklamanos I, et al. Hypersensitivity reactions associated with platinum antineoplastic agents: a systematic review. Met Based Drugs. 2010;: 207084

32. Lee C, Gianos M, Klaustermeyer WB. Diagnosis and management of hypersensitivity reactions related to common cancer chemotherapy agents. Ann Allergy Asthma Immunol. 2009; 102: 179-187. 